\title{
On the Impact of Premature Termination of Chemotherapy on Relapse of Breast Cancer
}

\author{
Heidrun Männle ${ }^{1^{*}}$, Karsten Münstedt ${ }^{1}$, Felix Momm ${ }^{2}$ and Jan Willem Siebers ${ }^{1}$ \\ ${ }^{1}$ Department of Gynaecology and Obstetrics, Ortenau-Klinikum Offenburg-Kehl, Germany
}

${ }^{2}$ Department of Radio-Oncology, Ortenau-Klinikum Offenburg-Kehl, Germany

*Corresponding author: Heidrun Männle, Department of Gynaecology and Obstetrics, Ortenau Klinikum Offenburg-Kehl, Ebertplatz 12, 77654 Offenburg, Germany, Tel: 0049/781-4723501, Fax: 0049/781-4723502

\begin{abstract}
Background: Some breast cancer patients receive a recommendation for adjuvant chemotherapy if necessary. In some cases, patients refuse this recommendation. In other cases, they start with chemotherapy, but then discontinue it. The question raised here are the effects of patients' rejection or premature termination of recommended chemotherapies.
\end{abstract}

Methods: 1916 patients, who received a recommendation for chemotherapy, were analyzed between 1997 and 2017. The analyses included descriptive analyses and Kaplan-Meier survival analyses.

Results: A percentage of the patients stopped prematurely $(8.4 \%)$ or totally refused adjuvant chemotherapy $(7.2 \%)$. They differed from those who completed the recommended chemotherapy with respect to age $(F=82.483 ; p<0.001$; ONEWAY-ANOVA), HER2-status (chi2 = 8.290; $\mathrm{df}=2 ; \mathrm{p}=$ 0.016 ), nodal status (chi2 $=12.785 ; \mathrm{df}=6 ; \mathrm{p}=0.047$ ) and type of surgery (breast conserving vs. mastectomy) (chi2 $=6.267 ; \mathrm{df}=2 ; \mathrm{p}=0.044)$. The Kaplan Meier disease-free survival analyses showed significant differences between these groups (Log RankDFS $=21.012 ; \mathrm{df}=2 ; \mathrm{p}<0.001$ ) . A significant influence on the overall survival was found in the subgroup of hormone-receptor-positive/HER2-negative patients (Log RankHR positive $=4.728 ; d f=1 ; p=0.030$ ).

Conclusions: Disease-free and disease-specific overall survival rates worsened in the subgroup of patients with hormone receptor positive/HER2 negative tumors if chemotherapy was stopped prematurely. It seems important to assess the patients' reasons for discontinuing, to emphasize the importance of chemotherapy and to explicitly encourage patients in this group to see it through to the very end.

\section{Keywords}

Breast cancer, Refusal chemotherapy, Premature termination

\section{Introduction}

In Germany breast cancer is by far the most common cancer in women, with approximately 69.000 new cases and 18.000 deaths each year [1]. Nevertheless, if detected early enough, breast cancer is a highly curable condition when current conventional treatment modalities such as surgery, radiation, and adjuvant drug treatment are employed. The drug treatment includes endocrine therapy, chemotherapy, or targeted therapy, depending on the biologic characteristics of the tumor. The recommendations for the treatment of breast cancer are based upon the national guidelines, which are likewise based mainly on the meta-analyses on various aspects of breast cancer treatment. These show that current treatment concepts lead to improved chances of overall survival, disease-free survival as well as local control [2-6]. In general, therapy recommendations are made by multidisciplinary breast tumor boards which consider and account for the individual patient characteristics, like comorbidity.

According to the German S3 guideline there is an indication for adjuvant combination chemotherapy for:

- HER2-positive tumors (from pT1b, N0; pT1a, NO if further risk: G3, ER / PR neg., Ki-67 high).

- Triple-negative tumors (ER- and PR-negative, HER2-negative).

- Luminal-B tumors with a high risk of recurrence (Ki-67 high, G3, high risk multigene assay, young age, lymph node involvement [7].

Citation: Männle H, Münstedt K, Momm F, Siebers JW, (2021) On the Impact of Premature Termination of Chemotherapy on Relapse of Breast Cancer. Int J Oncol Res 4:028. doi.org/10.23937/26434563/1710028

Accepted: March 13, 2021: Published: March 15, 2021

Copyright: (c) 2021 Männle $\mathrm{H}$, et al. This is an open-access article distributed under the terms of the Creative Commons Attribution License, which permits unrestricted use, distribution, and reproduction in any medium, provided the original author and source are credited. 
One principle of treatment is combination chemotherapy which aims to spread the side effects of the drugs, to reach the different cell lines in heterogeneous tumors and to prevent the development of resistant cell clones. Common chemotherapy regimens include taxanes and anthracyclines [8]. In trials in which taxanes were added to a fixed anthracycline regimen, thereby extending the duration of treatment, breast cancer mortality decreased (RR 0.86, SE 0.04 , bilateral significance $[2 \mathrm{p}]=0.0005)$ [5]. Another principle is that chemotherapy should be administered at the recommended doses. When lower dose intensities (reduced doses or prolonged intervals between courses) are administered, there is a risk of loss of effectiveness $[9,10]$. Prolonged adjuvant chemotherapy such as sequential anthracycline-taxane chemotherapy improves disease-free survival [11]. From the various trials on the subject it can be assumed that the omission of an effective medication or a dose reduction can lead to impaired effectiveness of the combination therapy and thus reduced chances for cure [12].

However, in some cases patients will decide against the tumor board's recommendation for chemotherapy, or discontinue it during the course. The reasons for this vary. Citrin, et al. demonstrated key factors in their trial for patients facing the decision to reject potentially life-prolonging conventional therapy. These included e.g. negative first experiences with "uncaring, insensitive, and unnecessarily harsh" oncologists, the fear of side effects, and a belief in the efficacy of alternative therapies [13]. Factors influencing physicians to stop chemotherapy early are essentially intolerable side effects, such as severe polyneuropathy, worsening of general health during chemotherapy and hematological problems (such as severe leucopenia or thrombocytopenia). However, the question arises as to the consequences of discontinuing a recommended chemotherapy too early. In order to answer this question, we analyzed the data base of the center for breast cancer at the Ortenau Clinic Offenburg, Germany.

\section{Material and Methods}

\section{Patients}

All the cases of non-metastatic primary breast cancer at the breast cancer center of the Ortenau Clinic Offenburg were analyzed, who received the recommendation for adjuvant chemotherapy from our multidisciplinary tumor board between 1997 and 2017. The recommendation as to whether a patient should receive chemotherapy was always given by an interdisciplinary tumor board according to the indications valid at the time of treatment. Patients with neoadjuvant chemotherapy were excluded because discontinuing neoadjuvant chemotherapy was mostly due to a lack of response and the patients received post-neoadjuvant chemotherapy. The aim of this work was to analyze whether the relevant discontinuations of adjuvant chemotherapy had an im- pact on patient survival. We considered chemotherapy as being completed if a patient received more than $80 \%$ of their recommended number of chemotherapy courses. If patients received less than or equal to $80 \%$ of the recommended courses, the treatments were rated as a relevant protocol violation.

\section{Tumor documentation system}

Our tumor documentation program MaDos covers various aspects of the tumor biology, the treatment and the follow up. This allows the complete evaluation of the management regarding the patients' treatment. The system also assessed if patients decided not to follow the advice for adjuvant chemotherapy treatment from the multidisciplinary breast tumor board or if there was a premature termination of the chemotherapy.

\section{Data management and statistics}

All data of the tumor documentation system MaDos were exported in Microsoft Excel 2010. These data were then transferred to IBM SPSS Statistics for Windows (version 24) for further statistical analyses. The analyses included descriptive analyses and Kaplan-Meier survival analyses.

\section{Ethics}

We obtained positive consent from the Ethics Committee of the Medical Faculty of the University of Freiburg i.Br. (application number 291/20).

\section{Results}

Among 3705 patients with non-metastatic primary breast cancer our multidisciplinary tumor board recommended chemotherapy in 1916 cases. 419 patients received a recommendation for primary chemotherapy, 1589 received adjuvant or post-neoadjuvant chemotherapy. Among these 1456 (91.6\%) patients completed relevant courses (more than $80 \%$ ) of chemotherapy. 133 (8.4\%) patients discontinued chemotherapy early. $7.2 \%$ $(138 / 1916)$ of the patients with recommended chemotherapies totally refused primary or adjuvant chemotherapy when they received the recommendation.

The characteristics of the entire group of patients, the subgroup of patients who completed more than $80 \%$ of adjuvant chemotherapy, the subgroup of those who discontinued chemotherapy early and those who refused chemotherapy are summarized in Table 1. When analyzing for tumor size, tumor stage $(T)$, nodal stage $(\mathrm{N})$ and the number of affected lymph nodes, we excluded patients with primary chemotherapy, as the above-mentioned values changed, and a bias would have taken place. Table 1 shows that patients who totally refused chemotherapy were significantly older $(F=$ 82.483; $p<.001$; ONEWAY-ANOVA) and had a negative nodal status more often $\left(\right.$ chi $\left.^{2}=12.785 ; d f=6 ; p=0.047\right)$. Patients who underwent a mastectomy were also more likely to reject chemotherapy $\left(\mathrm{chi}^{2}=6.267 ; \mathrm{df}=2 ; \mathrm{p}=\right.$ 
Table 1: Patients' characteristics.

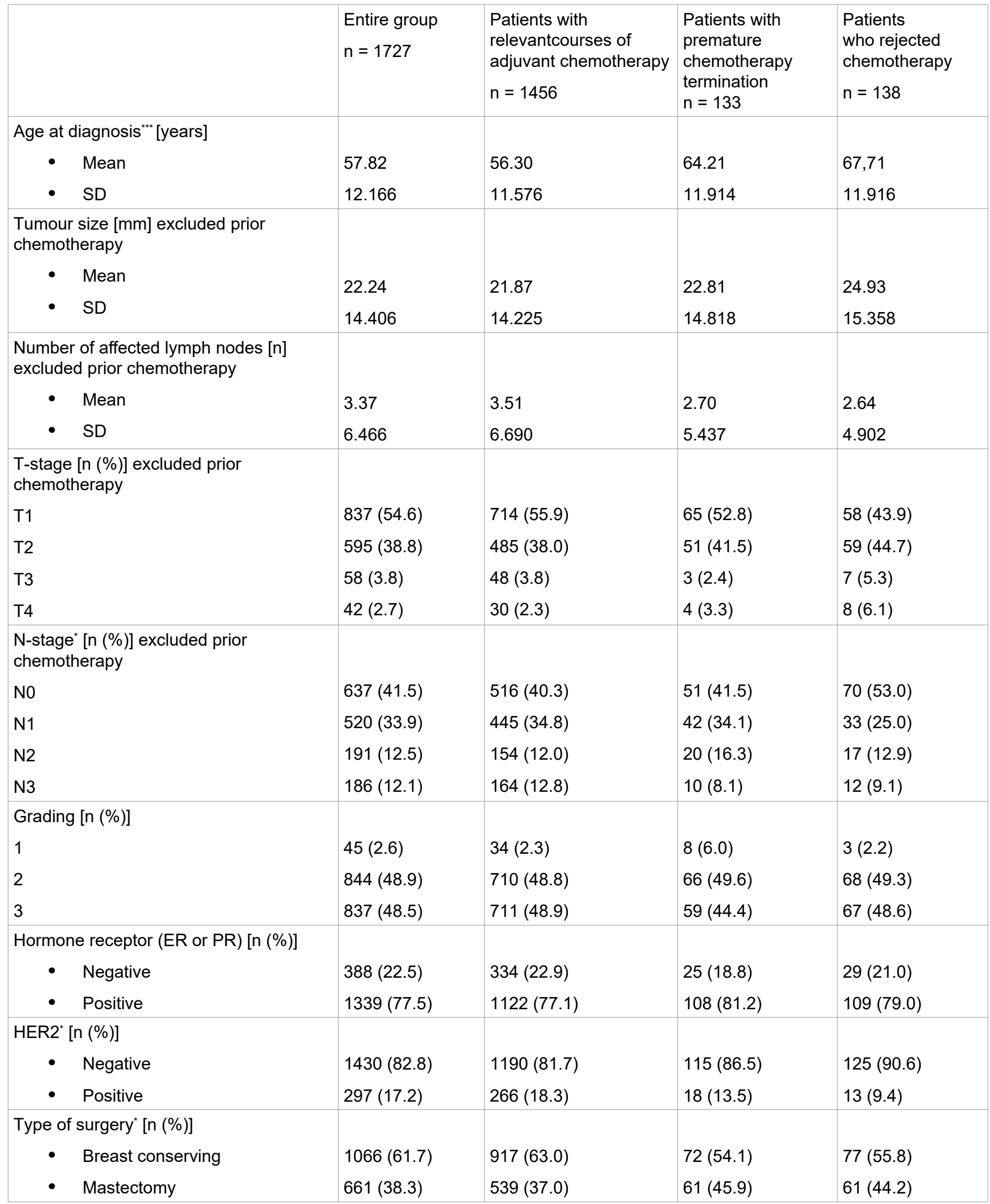

${ }^{*}=p<0.05 ;{ }^{* *}=p<0.01 ;{ }^{* * *}=p<0.001$

Characteristics of the entire group of patients, the subgroup of patients who completed more than $80 \%$ of adjuvant chemotherapy and the subgroup of those with early discontinuation of chemotherapy and those who refused chemotherapy are summarized in Table 1.

The differences between patients who did relevant cycles of chemotherapy, who premature stopped chemotherapy and those who disagreed are significantly different regarding age $\left(F=82.483 ; p<0.001\right.$; ONEWAY-ANOVA), Her2-status $\left(\right.$ chi ${ }^{2}=8.290 ;$ df $=2 ; p=0.016), \mathrm{N}$-stage $\left(\mathrm{chi}^{2}=12.785 ; \mathrm{df}=6 ; \mathrm{p}=0.047\right)$ and type of surgery (breast conserving vs. mastectomy) $\left(\right.$ chi ${ }^{2}=6.267 ;$ $\mathrm{df}=2 ; \mathrm{p}=0.044)$. 
0.044) too. More elderly patients and those who had had mastectomies discontinued chemotherapy earlier, too. Those who had a positive HER2 status were less

likely to reject or prematurely stop chemotherapy $\left(\mathrm{chi}^{2}\right.$ $=8.290 ; \mathrm{df}=2 ; \mathrm{p}=0.016$ ).

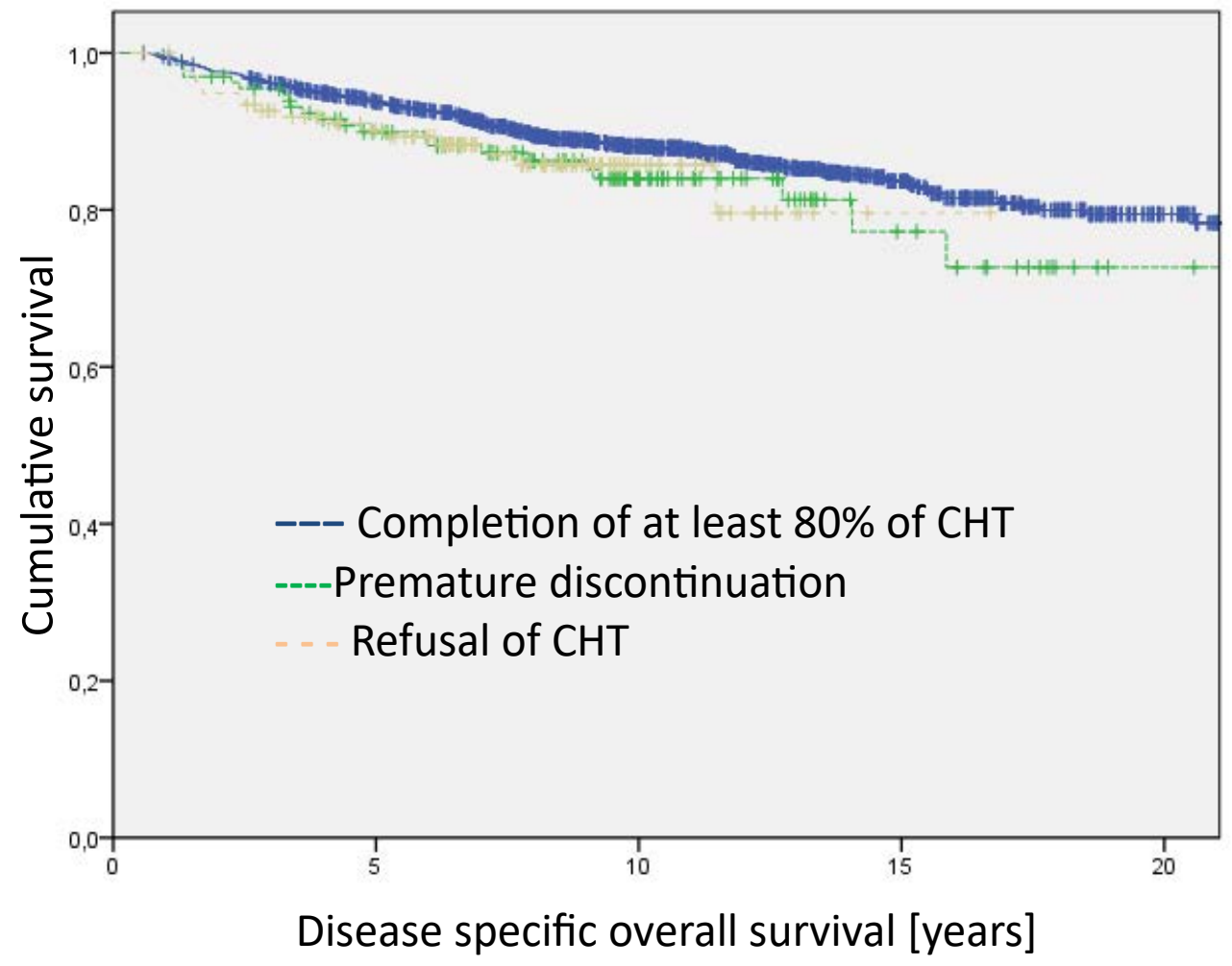

Figure 1: Analysis of the entire group regarding disease specific overall survival. The differences between the groups are not significant. The median survival time of the groups are 20.4 years for Completion of at least $80 \%$ of $\mathrm{CHT}, 18.5$ years for Premature discontinuation and 14.5 for Refusal of $\mathrm{CHT}$.

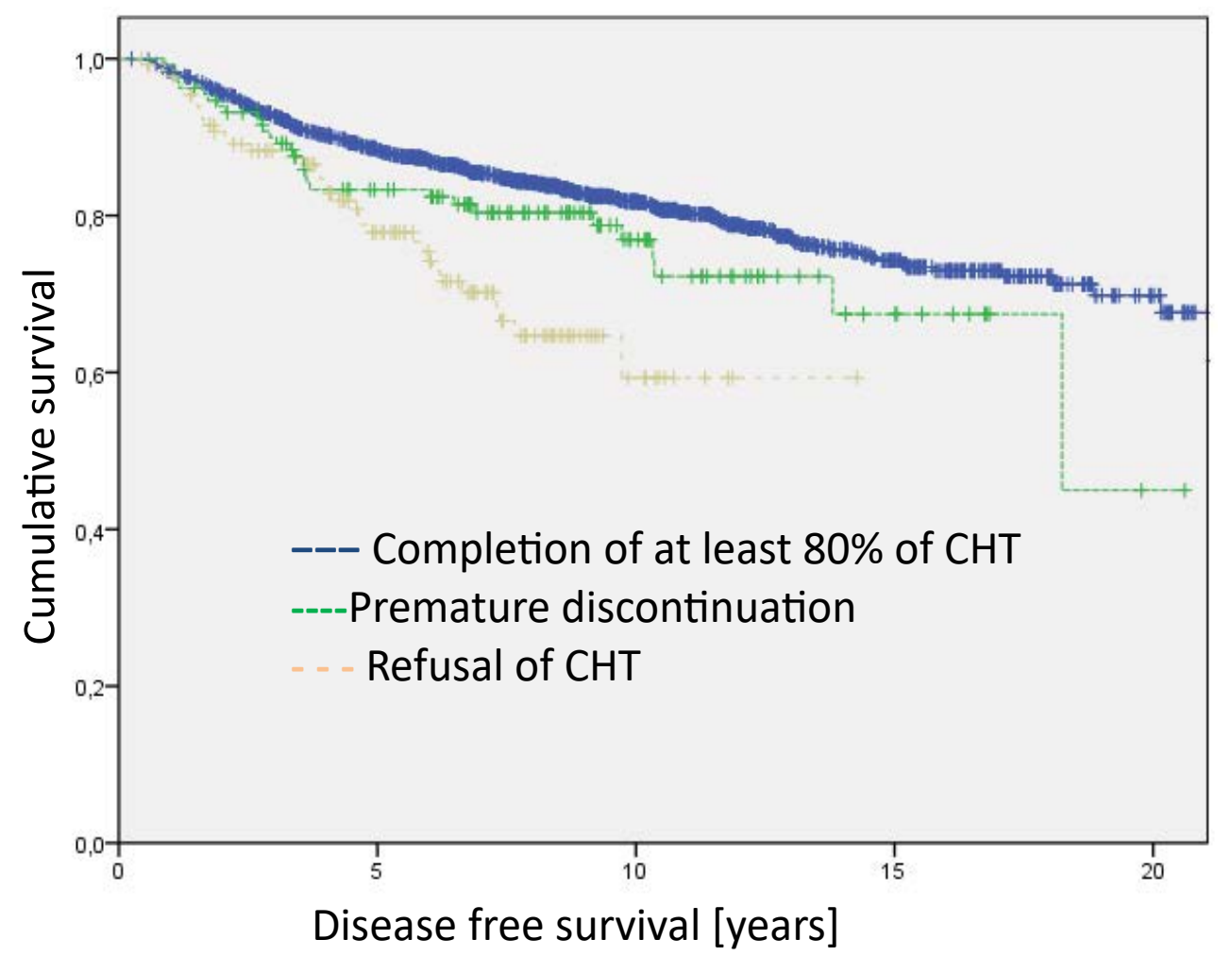

Figure 2: Analysis of the entire group regarding disease free survival (Log Rank DFS $=21.012 ; \mathrm{df}=2 ; \mathrm{p}<0.001)$. The median survival time of the groups are 17.9 years for Completion of at least $80 \%$ of $\mathrm{CHT}, 15.4$ years for Premature discontinuation and 10.5 for Refusal of CHT. 
The Kaplan Meier disease-specific overall survival analyses did not show statistically significant differences between patients who completed the relevant cycles of chemotherapy and those who discontinued early in the treatment and those who totally refused chemotherapy (Figure 1). However, the analyses on disease-free survival revealed highly significant differences (Figure 2, Log Rank DFS = 21.012; df = 2; $<<0.001$ ).

Further analyses investigated the potential impact of additional therapies, e.g., endocrine therapy, or anti-HER2-therapy, on survival. Here, we compared the patients who had completed the relevant chemotherapy courses against those who had discontinued chemotherapy early. The patients were divided into "HER2-positive who also received anti-HER2-therapy", "hormone-receptor-positive/ HER2-negative who received endocrine therapy" and "triple-negative".

Regarding disease-specific overall survival, we found no differences in the subgroups of HER2-positive and triple negative breast cancer patients. However, we found significant differences in the hormone-receptor-positive/ HER2-negative subgroup favoring patients who completed relevant courses of chemotherapy (Figure 3, Log RankHR positive $=4.728 ; d f=1 ; p=0.030$ ). Similarly, the only subgroup with significant differences regarding disease-free survival were patients with hormone receptor positive/HER2 negative (Figure 4, Log Rank HR positive $=7.885 ; \mathrm{df}=1 ; \mathrm{p}=0.005$ ).

Further subgroup analyses on age with the sub- groups "< 50 years", "50 to 69 years" (because of the screening age in Germany) and " $\geq 70$ years" showed no statistically significant differences with respect to disease-free survival and disease-specific survival between patients discontinuing chemotherapy or those with relevant courses of chemotherapy. However, a tendency towards worse disease-free survival and disease-specific survival in the group of the " $<50$ years" was found when chemotherapy was discontinued early (Figure 5 and Figure 6).

\section{Discussion}

This study shows that substantial percentages of patients discontinued recommended chemotherapy prematurely or even rejected it altogether, refusing to even start with the treatment. Given that there was a greater percentage of an older woman in the collective of patients who had refused or stopped chemotherapy prematurely, it can be assumed that the fear of side effects could be an important differentiating factor among the collectives. In this respect, our study supports the findings of other studies $[14,15]$. Likewise, patients with nodal negative status and patients who had had mastectomies were more likely to reject the recommended chemotherapy. To the best of our knowledge, this is the first study to show that patients with a mastectomy or negative node status are more likely to reject chemotherapy. This could be related to the fact that patients do not clearly understand the need for chemotherapy even if lymph nodes are unaffected or after mastecto-

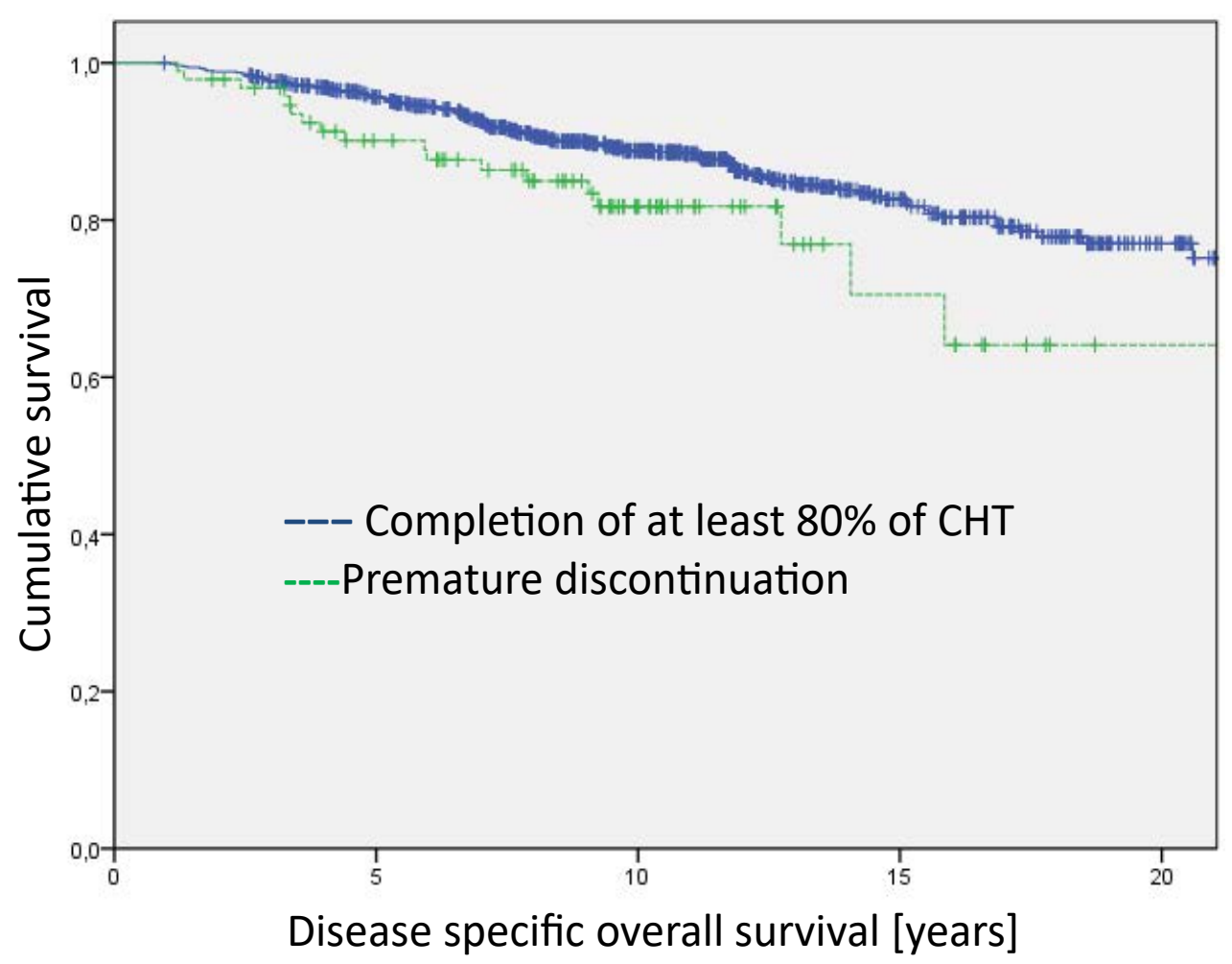

Figure 3: Analysis of the Hormone-receptor-postive/Her2-negative group regarding disease specific overall survival (Log Rank HR positive $=4.728 ; \mathrm{df}=1 ; p=0.030$ ). The median survival time of the groups are 20.3 years for Completion of at least $80 \%$ of $\mathrm{CHT}$ and 17.7 years for Premature discontinuation. 


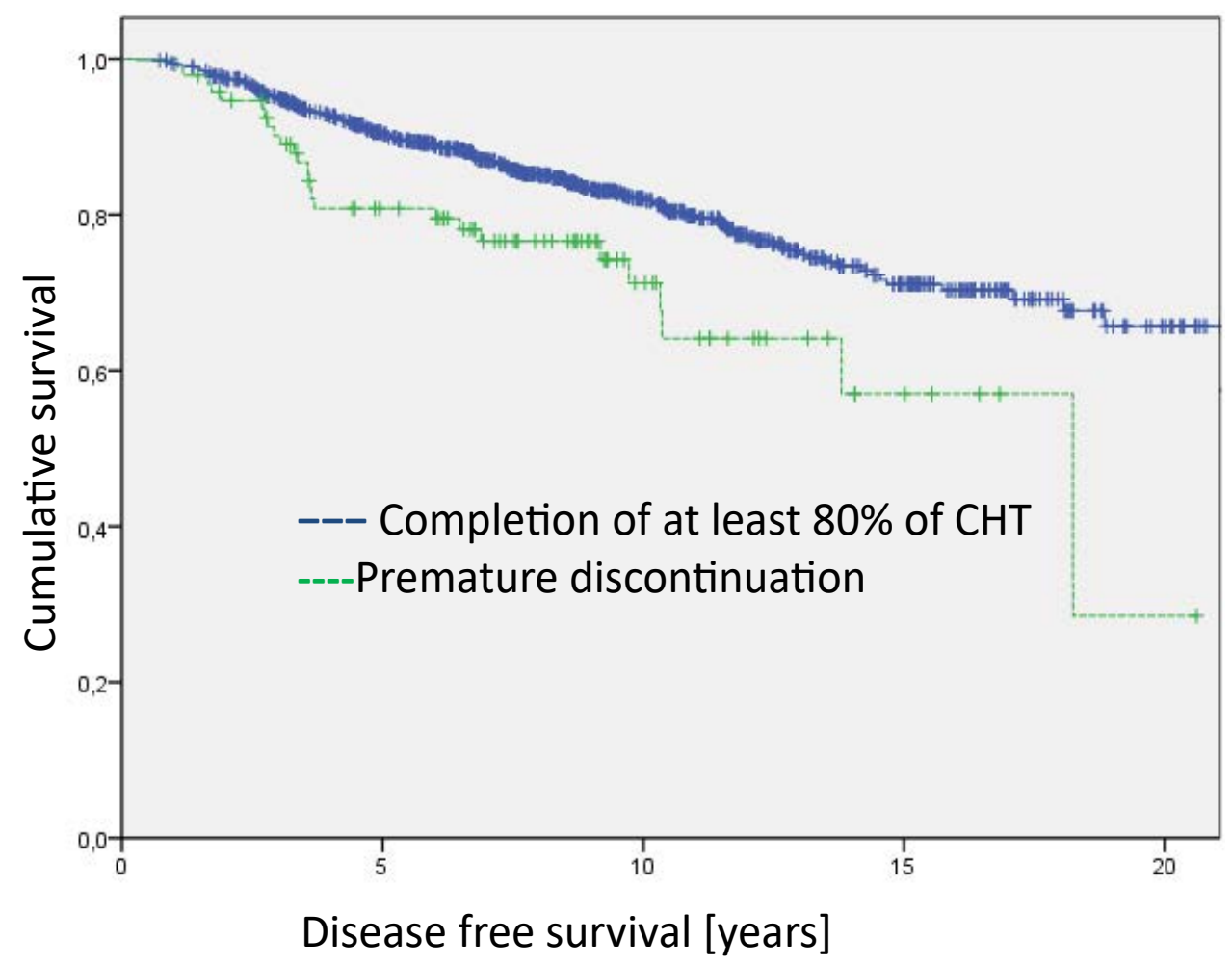

Figure 4: Analysis of the Hormone-receptor-postive/Her2-negative group regarding disease free survival (Log Rank HR positive $=7.885$; $\mathrm{df}=1 ; \mathrm{p}=0.005$ ). The median survival time of the groups is 17.8 years for Completion of at least $80 \%$ of $\mathrm{CHT}$ and 14.1 years for Premature discontinuation.

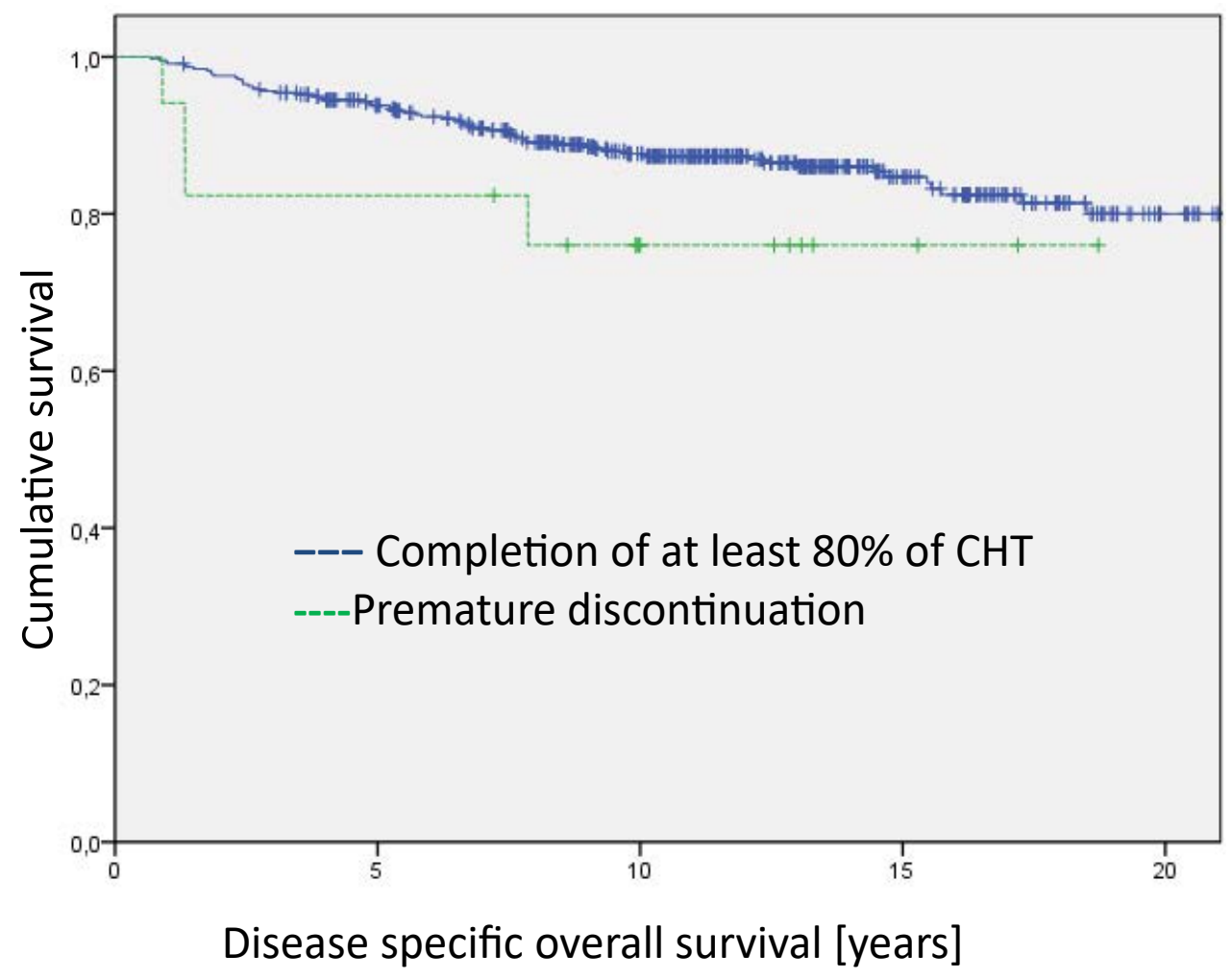

Figure 5: Analysis of the age < 50 years group regarding disease specific overall survival. The differences between the groups are not significant. The median survival time of the groups is 20.6 years for Completion of at least $80 \%$ of $\mathrm{CHT}$ and 14.9 years for Premature discontinuation. 


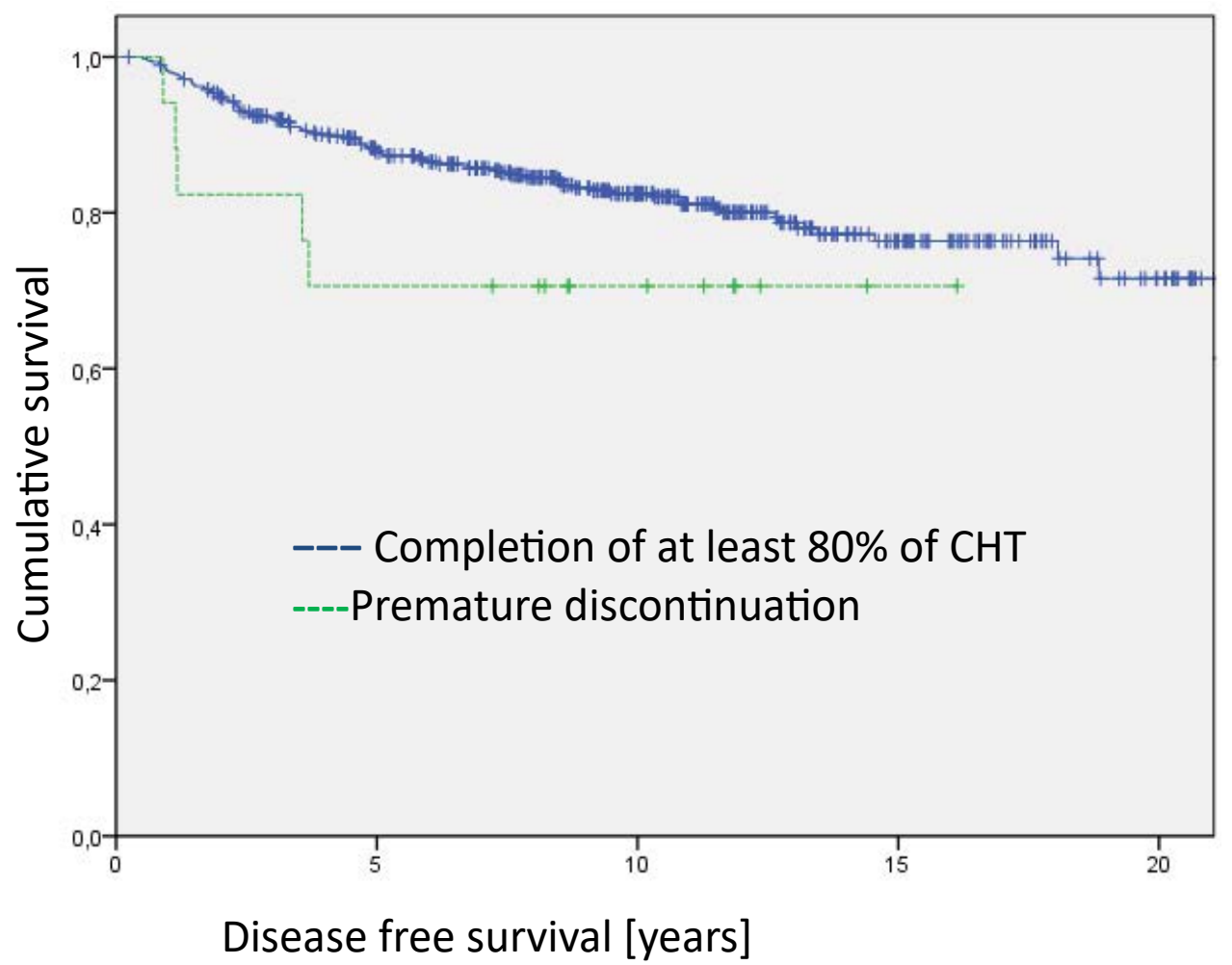

Figure 6: Analysis of the age $<50$ years group regarding disease free survival. The differences between the groups are not significant. The median survival time of the groups is 18.0 years for Completion of at least $80 \%$ of $\mathrm{CHT}$ and 12.0 years for Premature discontinuation.

my, believing that surgical therapy could be sufficient. Barak, et al. clearly showed disparities between the views of patients and doctors in regard to goals, chances of attainment, side effects, and difficulty of treatment [16] in their study too. In this regard, patient communication should be improved so that patients understand the reasoning behind the advice for adjuvant therapy. This is even more important as Johnson, et al. was able to show that patients who opted for complementary medicine (CM) and thus rejected the recommended standard therapies had a higher risk of cancer-associated death. In particular, it can be assumed that the rejection of chemotherapy and the increased mortality risk were related [17]. Patients in the CM group were more likely to be younger than those who did not use CM, be female, of a higher socioeconomic status and a higher educational level and have breast cancer or colorectal cancer. Unfortunately, the type of breast cancer surgery was not recorded in the referred study.

The analyses in this investigation also showed that early discontinuation of chemotherapy leads to poorer disease-free survival in general and worse disease-specific and disease-free survival in the subgroup of hormone receptor positive/Her2 negative patients. This is consistent with the data that shows that scheduled completion of chemotherapy is beneficial $[11,18]$.

The analysis of the subgroup "HER2 positive who also received anti-HER2 therapy" showed no difference in survival curves. This may be attributed to the effec- tiveness of the additional targeted anti-HER2 therapy, which was administered for an entire year. Also, the fact that HER2 positive patients carried out the chemotherapy as planned (Table 1) reflects that the risk of recurrence is realized by the patient and/or by the physician. However, it must be mentioned that there were relatively few cases that were recorded in the group of chemotherapy dropouts. These results are in accordance with Tolaney, et al. who showed that de-escalated chemotherapy with anti-HER2 therapy is particularly possible with small nodal-negative tumors. In their study, the patients received a course of weekly treatment with paclitaxel and trastuzumab for 12 weeks, followed by 9 months of trastuzumab monotherapy $[19,20]$. Burstein also showed in his overview on the anti-HER2-directed chemotherapy regimens that anthracycline-free chemotherapy can now be regarded as a standard [21].

The "triple negative" breast cancers (TNBC) also showed no differences in the survival curve. However, it must be recognized that we now know that TNBC is a very heterogeneous group. Molecular sub typing differentiates four subtypes using gene expression analyses - two basal-like subtypes, a subtype characterized by tumor-infiltrating lymphocytes and tumor-associated mesenchymal cells as well as a luminal type likely controlled via the androgen receptors [22]. Therefore, the TNBC group cannot be simply or easily compared, especially also since there were very few cases that were recorded under the chemotherapy dropout group. 
The group with the hormone receptor positive/ HER2-receptor negative tumors showed a significantly worse survival curve when chemotherapy was prematurely stopped. Other authors have also demonstrated that the patients in this group are at high risk with primary tumor sizes $(>2 \mathrm{~cm}$ ) and higher primary tumor grades (2/3) [23]. It is probably because they respond more slowly to chemotherapy and therefore need to fully complete all the planned cycles to compensate for the lower sensitivity over a longer period, as it has been shown in neoadjuvant chemotherapy trials $[24,25]$. In a phase two study, neoadjuvant endocrine therapy with Letrozole and Palbociclib was compared against chemotherapy. Although the authors of the referred study concluded that endocrine combination therapy is an alternative to chemotherapy, it was found that the remission rates were significantly lower under endocrine therapy [26]. Haque, et al. were able to show that chemotherapy response rates in luminal-B tumors are fairly low in many patients (less than 10\%). Nevertheless, the degree of response is associated with the overall survival, especially in patients with luminal-B, HER2 and triple-negative [25]. Thus the fact that these patients are suitable for endocrine therapy cannot be regarded as a reason for discontinuation of necessary chemotherapy. The increased risk due to the tumor stage or tumor biology cannot be compensated by endocrine therapy alone but must be minimized first by undergoing additional chemotherapy. Patients with luminal-B breast cancer require fully completed chemotherapy. So, it is even more important to perceive this group as a highrisk group and to encourage them to complete their chemotherapy through right to the end. These are the reasons why physicians have to motivate patients not to discontinue chemotherapy prematurely. As already mentioned, this requires time and a good relationship between physician and patient. This demand is supported by the results of the PACTOT-study [27].

There are some limitations to our study. One problem that the reasoning behind the treatment recommendations for patients who, despite tumor related risk factors, did not receive a chemotherapy recommendation was not assessed. Characteristics such as age, performance status, or a previous history of cancer could have played a major role. It would be interesting to compare the tumor-related survival curves of these patients with the patients who refused chemotherapy or discontinued during the course.

Another shortcoming of this work is that the reasons why chemotherapy was prematurely stopped were not recorded in detail. Thus it remains unclear, whether this happened for medical reasons or upon patient's request.

A consequence of our results is the apparent necessity of informing patients on the importance of fully dosed chemotherapy. It is questionable as to whether this can be realized under real-life conditions in Germany. However, considering the consequences it appears to be of great importance. Health care providers must provide the means for the individual consultation of patients regarding the importance of chemotherapy. This concept is supported by a study by Riese, et al. who were able to show how important individualized care is in the context of chemotherapy [27].

An "Onko-Coach" was developed as part of the PACTOT-study to evaluate the impact of a standardized patient education program provided by specially trained oncology nurses on therapy management regarding the side effects and unplanned therapy interruptions. The patients of the intervention group reported fewer side effects and less interruptions of the therapy compared to the control group [27].

\section{Conclusions}

This study shows that patients with nodal negative status and patients who underwent a mastectomy were more likely to reject the recommended chemotherapy. In this regard, patient communication should be improved so that patients understand the reasoning behind the advice for adjuvant therapy. Likewise, it was shown that the discontinuation of chemotherapy is a relevant problem. This does not necessarily lead to worsened disease-specific overall survival but to worse disease-free survival. Patients in some subgroups (HR-positive/HER2 negative) are at greater risk. Here it appears important to advise these patients to adhere to the recommended treatment protocols. If side effects should be the underlying reason, all effort should be made to reduce them as well as possible using reasonable concepts from supportive care to eventually considering concepts from integrative medicine. Also, the assumption that these patients can be treated just as well with endocrine therapy should not lead to the indicated chemotherapy being carelessly discontinued.

\section{Acknowledgement}

We would like to thank all those who have contributed to the establishment and maintenance of our data bank on breast cancer. This includes our tumor-documenters, namely Mrs. Andrea Heuberger, as well as all the gynecologists and general practitioners in our region who supplied us with information about the follow-up of the patients. We would especially like to thank Mr. Alexander Schattenberg, who developed the MaDos documentation system and was always ready to assist us with special queries.

\section{Statement of Ethics}

Authors state that subjects (or their parents or guardians) have given their written informed consent.

The consent for the project was given by the ethics committee of the University of Freiburg (application number 291/20). 


\section{Conflict of Interest Statement}

All authors declare no conflict of interest.

\section{Sources of Support}

This research did not receive any specific grant from any funding agencies in the public, commercial, or notfor-profit sectors.

\section{Statement of Equal Authors' Contribution}

Dr. Heidrun Männle: Original idea, planning, and input of the manuscript, Data collection, Analysis and interpretation of data, Writing the manuscript.

Prof. Dr. Karsten Münstedt: Reading/commenting/ editing of manuscript, Critical revision of the manuscript, Supervision of the study/manuscript, Training/ education of authors, Mentoring of $1^{\text {st }}$ author, Statistical Expertise.

Prof. Dr. Felix Momm: Reading/commenting/editing of manuscript, Critical revision of the manuscript.

Prof. Dr. Jan Willem Siebers: Reading/commenting/ editing of manuscript, Supervision of the study/manuscript, Training/education of authors, Mentoring of $1^{\text {st }}$ author.

\section{References}

1. https://www.krebsdaten.de/Krebs/DE/Content/Krebsarten/ Brustkrebs/brustkrebs_node.html

2. Darby S, McGale P, Correa C, Taylor C, Arriagada R, et al. (2011) Effect of radiotherapy after breast-conserving surgery on 10-year recurrence and 15-year breast cancer death: Meta-analysis of individual patient data for 10,801 women in 17 randomised trials. Lancet 378: 1707-1716.

3. Davies C, Godwin J, Gray R, Clarke M, Cutter D, et al. (2011) Relevance of breast cancer hormone receptors and other factors to the efficacy of adjuvant tamoxifen: Patient-level meta-analysis of randomised trials. Lancet 378 : 771-784.

4. McGale P, Taylor C, Correa C, Cutter D, Duane F, et al. (2014) Effect of radiotherapy after mastectomy and axillary surgery on 10-year recurrence and 20-year breast cancer mortality: Meta-analysis of individual patient data for 8135 women in 22 randomised trials. Lancet 383: 2127-2135.

5. Peto R, Davies C, Godwin J, Gray R, Pan HC, et al. (2012) Comparisons between different polychemotherapy regimens for early breast cancer: Meta-analyses of long-term outcome among 100,000 women in 123 randomised trials. Lancet 379: 432-444.

6. Early Breast Cancer Trialists' Collaborative Group (2018) Long-term outcomes for neoadjuvant versus adjuvant chemotherapy in early breast cancer: meta-analysis of individual patient data from ten randomised trials. Lancet Oncol 19: 27-39.

7. Interdisziplinäre (2020) S3-Leitlinie für die Früherkennung, Diagnostik, Therapie und Nachsorge des Mammakarzinoms. Leitlinienprogramm Onkologie 032-045OL.

8. Estévez LG, Muñoz M, Alvarez I, Fernández Y, García-Mata J, et al. (2007) Evidence-based use of taxanes in the adjuvant setting of breast cancer. A review of randomized phase III trials. Cancer Treat Rev 33: 474-483.
9. Fumoleau $P$, Kerbrat $P$, Romestaing $P$, Fargeot $P$, Brémond $A$, et al. (2003) Randomized trial comparing six versus three cycles of epirubicin-based adjuvant chemotherapy in premenopausal, node-positive breast cancer patients: 10-year follow-up results of the French Adjuvant Study Group 01 trial. J Clin Oncol 21: 298-305.

10. French Adjuvant Study Group (2001) Benefit of a high-dose epirubicin regimen in adjuvant chemotherapy for node-positive breast cancer patients with poor prognostic factors: 5-year follow-up results of French Adjuvant Study Group 05 randomized trial. J Clin Oncol 19: 602-611.

11. Swain SM, Jeong JH, Geyer CE Jr, Costantino JP, Pajon ER, et al. (2010) Longer therapy, iatrogenic amenorrhea, and survival in early breast cancer. $\mathrm{N}$ Engl J Med 362: 2053-2065.

12. DeVita, Jr. Vincent T (2003) Principles of chemotherapy. In: Jr. Vincent T, Hellmam Samuel, Rosenberg Steven A. DeVita, Cancer, principles \& practice of oncology. ( $4^{\text {th }}$ edn $)$, Lippincott Company.

13. Citrin DL, Bloom DL, Grutsch JF, Mortensen SJ, Lis CG (2012) Beliefs and Perceptions of Women with Newly Diagnosed Breast Cancer Who Refused Conventional Treatment in Favor of Alternative Therapies. Oncologist 17: 607612.

14. Marcinkowski EF, Ottesen R, Niland J, Vito C (2017) Acceptance of adjuvant chemotherapy recommendations in early-stage hormone-positive breast cancer. J Surg Res 214: 79-85.

15. Ma AM, Barone J, Wallis AE, Wu NJ, Garcia LB, et al. (2008) Noncompliance with adjuvant radiation, chemotherapy, or hormonal therapy in breast cancer patients. Am J Surg 196: 500-504.

16. Barak F, Ostrowsky LA, Kreitler S (2012) Adjuvant chemotherapy for breast cancer patients: Patients' expectations and physicians' attitudes. Palliat Support Care 10: 107-114.

17. Johnson SB, Park HS, Gross CP, Yu JB (2018) Complementary Medicine, Refusal of Conventional Cancer Therapy, and Survival Among Patients With Curable Cancers. JAMA Oncol 4: 1375-1381.

18. Henderson IC, Berry DA, Demetri GD, Cirrincione CT, Goldstein LJ, et al. (2003) Improved outcomes from adding sequential Paclitaxel but not from escalating Doxorubicin dose in an adjuvant chemotherapy regimen for patients with node-positive primary breast cancer. J Clin Oncol 21: 976-983.

19. Tolaney SM, Barry WT, Dang CT, Yardley DA, Moy B, et al. (2015) Adjuvant paclitaxel and trastuzumab for node-negative, HER2-positive breast cancer. N Engl J Med 372: 134141.

20. Tolaney SM, Guo H, Pernas S, Barry WT, Dillon DA, et al. (2019) Seven-Year Follow-Up Analysis of Adjuvant Paclitaxel and Trastuzumab Trial for Node-Negative, Human Epidermal Growth Factor Receptor 2-Positive Breast Cancer. J Clin Oncol 37: 1868-1875.

21. Burstein HJ, Piccart-Gebhart MJ, Perez EA, Hortobagyi GN, Wolmark N, et al. (2012) Choosing the Best Trastuzumab-Based Adjuvant Chemotherapy Regimen: Should We Abandon Anthracyclines? J Clin Oncol 18: 2179-2182.

22. Lehmann BD, Jovanović B, Chen X, Estrada MV, Johnson KN, et al. (2016) Refinement of Triple-Negative Breast Cancer Molecular Subtypes: Implications for Neoadjuvant Chemotherapy Selection. PLoS One 11: e0157368.

23. Yu CC, Kuo WL, Shen SC, Chou HH, Lo YF, et al. (2020) 
Prognostic study for isolated local recurrence operated with salvage excision in hormone-receptor-positive patients with invasive breast cancer after primary breast surgery. Biomed J 43: 83-93.

24. Rouzier R, Perou CM, Symmans WF, Ibrahim N, Cristofanilli $\mathrm{M}$, et al. (2005) Breast cancer molecular subtypes respond differently to preoperative chemotherapy. Clin Cancer Res 11: 5678-5685.

25. Haque W, Verma V, Hatch S, Klimberg VS, Butler EB, et al. (2018) Response rates and pathologic complete response by breast cancer molecular subtype following neoadjuvant chemotherapy. Breast Cancer Res Treat 170: 559-567.
26. Cottu P, D'Hondt V, Dureau S, Lerebours F, Desmoulins I, et al. (2018) Letrozole and palbociclib versus chemotherapy as neoadjuvant therapy of high-risk luminal breast cancer. Ann Oncol 29: 2334-2340.

27. Riese C, Weiß B, Borges U Jr, Beylich A, Dengler R, et al. (2017) Effectiveness of a standardized patient education program on therapy-related side effects and unplanned therapy interruptions in oral cancer therapy: A cluster-randomized controlled trial. Support Care Cancer 25: 34753483. 